\title{
Optical transfer function expansion of quadratic pupils
}

Jim Schwiegerling

Jim Schwiegerling, "Optical transfer function expansion of quadratic pupils," Proc. SPIE 10590, International Optical Design Conference 2017, 1059005 (27 November 2017); doi: 10.1117/12.2286284

SDIE Event: International Optical Design Conference - IODC 2017, 2017, Denver, United States 


\title{
Optical Transfer Function Expansion of Quadratic Pupils
}

\author{
Jim Schwiegerling \\ University of Arizona, 1630 E University Blvd, Tucson, Arizona 85721 \\ jschwieg@u.arizona.edu
}

\begin{abstract}
Quadratic pupils representing Gaussian apodization and defocus are expanded into Zernike polynomials. Combinations of the pupil expansion coefficients are used, in turn to expand the Optical Transfer Function into a novel set of basis functions.
\end{abstract}

Keywords: Optical Transfer Functions, Aberrations, Apodization.

\section{Introduction}

The Optical Transfer Function(OTF) is a versatile metric of the performance of optical systems. In earlier research, a technique for decomposing the complex pupil function of the system into Zernike polynomials was developed. The expansion coefficients (and their complex conjugates) for this pupil function decomposition were then used to form a linear expansion of the OTF into a novel set of basis functions.[1-3] The advantage of this technique is that the complex pupil function is used so that both apodization and wavefront error can be assessed and that the form of the pupil function is directly connected to the components of the OTF. This technique has applications in aberration theory, lens design and optimization. Here, the pupil function is restricted to having a quadratic form, meaning that only Gaussian apodization in the presence of defocus is considered. The pupil decomposition coefficients and subsequently the OTF expansion can be solved analytically. For useful apodization and defocus levels, these expansions also converge rapidly.

\section{Methods}

The pupil function $P(\rho)$ of a rotationally symmetric optical system is in general a complex function. Here, $\rho$ is the normalized radial coordinate. The magnitude of the pupil function describes the pupil apodization or transmission as a function of position. The argument of the pupil function describes the wavefront error of the system. The OTF for rotationally symmetric systems was first described in [1]. The techniques were then expanded to the non-rotationally symmetric case in [2-3]. Here, a subset of rotationally symmetric pupil functions with the form

$$
P(\rho)=\operatorname{cyl}\left(\frac{\rho}{2}\right) \exp \left(i a \rho^{2}\right),
$$

where $\operatorname{cyl}(\mathrm{)})$ is the cylinder function defining the unit pupil and $a=-2 \pi W_{20}+i \alpha_{20}$, is a complex constant. The constant $W_{20}$ is the amount of defocus in units of waves in the system. The constant $a_{20}$ is the Gaussian apodization factor. Following [1], the pupil function is decomposed into a set of rotationally symmetric Zernike radial polynomials such that

$$
P(\rho)=\sum_{n=0}^{N} b_{n 0} R_{n}^{0}(\rho)
$$

where $b_{n 0}$ are the expansion coefficients, $R_{n}^{0}(\rho)$ are the rotationally symmetric Zernike radial polynomials and $\mathrm{N}$ is the maximum order of the polynomials.[4] The expansion coefficients are then given by

$$
b_{n 0}=2(n+1) \int_{0}^{1} P(\rho) R_{n}^{0}(\rho) \rho d \rho .
$$

The expansion coefficients $b_{n 0}$ are in general complex valued. The index $n$ must be even due to the constraints on the Zernike Radial polynomials. The linear expansion of the OTF is then given by

$$
\operatorname{OTF}(\beta)=\frac{1}{c_{N}} \sum_{\substack{n=0 \\ n \text { even }}}^{N} \sum_{\substack{n^{\prime}=0 \\ n^{\prime} \text { even }}}^{N}(-1)^{n} b_{n 0} b_{n^{\prime} 0}^{*} G_{n+1, n^{\prime}+1,0}(\beta),
$$

International Optical Design Conference 2017, edited by Peter P. Clark, Julius A. Muschaweck, Richard N. Pfisterer, John R. Rogers, Proc. of SPIE-OSA Vol. 10590, 1059005 · @ 2017 SPIE

CCC code: $0277-786 X / 17 / \$ 18 \cdot$ doi: $10.1117 / 12.2286284$ 
where $\mathrm{c}_{\mathrm{N}}=\sum_{\text {even } \mathrm{n}}^{\mathrm{N}}\left|\mathrm{b}_{\mathrm{n} 0}\right|^{2} /(\mathrm{n}+1)$ and $G_{n+1, n^{\prime}+1,0}(\beta)$ are a set of rotationally symmetric basis functions (G-Functions) derived in [1]. Note, in previous reports, these were described in the form $G_{n+1, n^{\prime}+1,0}(-1, \beta)$. Here, the -1 in the argument will be dropped since it is only an artifact of the derivation of these functions. Table 1 summarizes the first few rotationally symmetric GFunctions. The preceding equations illustrate that the pupil function can be decomposed into a linear expansion with coefficients $b_{n 0}$. The OTF is then described by a linear expansion in terms of G-Functions with expansion coefficients which are simply the products of the various $b_{n 0}$ and their complex conjugates.

Table 1. Rotationally symmetric G-Functions arising for quadratic pupils up to $5^{\text {th }}$ order.

\begin{tabular}{|c|r|}
\hline$G_{110}(\beta)$ & $\frac{2}{\pi}\left[\cos ^{-1}\left(\frac{\beta}{2}\right)-\left(\frac{\beta}{2}\right) \sqrt{1-\frac{\beta^{2}}{4}}\right]$ \\
\hline$G_{130}(\beta)$ & $\frac{1}{\pi}\left[-2 \beta^{2} \cos ^{-1}\left(\frac{\beta}{2}\right)+\left(\beta^{2}+4\right)\left(\frac{\beta}{2}\right) \sqrt{1-\frac{\beta^{2}}{4}}\right]$ \\
\hline$G_{330}(\beta)$ & $\frac{1}{\pi}\left[\frac{2}{3} \cos ^{-1}\left(\frac{\beta}{2}\right)+\left(-\frac{2 \beta^{4}}{9}+\frac{14 \beta^{2}}{9}-\frac{10}{3}\right)\left(\frac{\beta}{2}\right) \sqrt{\left.1-\frac{\beta^{2}}{4}\right]}\right]$ \\
\hline$G_{150}(\beta)$ & $\frac{1}{\pi}\left[\left(6 \beta^{2}+6 \beta^{4}\right) \cos ^{-1}\left(\frac{\beta}{2}\right)+\left(-\frac{4 \beta^{4}}{3}+\frac{71 \beta^{2}}{3}-4\right)\left(\frac{\beta}{2}\right) \sqrt{1-\frac{\beta^{2}}{4}}\right]$ \\
\hline$G_{350}(\beta)$ & $\frac{1}{\pi}\left[-4 \beta^{2} \cos ^{-1}\left(\frac{\beta}{2}\right)+\left(\frac{\beta^{6}}{6}-\frac{5 \beta^{4}}{3}+7 \beta^{2}+4\right)\left(\frac{\beta}{2}\right) \sqrt{1-\frac{\beta^{2}}{4}}\right]$ \\
\hline$G_{550}(\beta)$ & $\frac{1}{\pi}\left[\frac{2}{5} \cos ^{-1}\left(\frac{\beta}{2}\right)+\left(-\frac{2 \beta^{8}}{25}+\frac{21 \beta^{6}}{25}-\frac{236 \beta^{4}}{75}+\frac{76 \beta^{2}}{15}-\frac{18}{5}\right)\left(\frac{\beta}{2}\right) \sqrt{1-\frac{\beta^{2}}{4}}\right]$ \\
\hline
\end{tabular}

The factor $c_{N}$ in Eq. 5 is useful as well, as it converges to the relative transmission of the pupil. This factor enables the number of terms $N$ in the expansions required for convergence. In the non-apodized case, $c_{N}$ will converge to unity. When Gaussian apodization is present, the factor $c_{N}$ will converge to

$$
c_{N} \Rightarrow 2 \int_{0}^{1}\left|\exp \left(-\alpha_{20} \rho^{2}-i 2 \pi W_{20} \rho^{2}\right)\right|^{2} \rho d \rho=\frac{1-\exp \left(-2 \alpha_{20}\right)}{2 \alpha_{20}} .
$$

The expansion coefficients $b_{n 0}$ for a quadratic pupil can be solved for analytically. Boivin [5] gives

$$
\exp \left(i a \rho^{2}\right)=\exp \left(\frac{i a}{2}\right) \sqrt{\frac{\pi}{a}} \sum_{s=0}^{\infty}(2 s+1) i^{s} J_{s+1 / 2}\left(\frac{a}{2}\right) R_{2 s}^{0}(\rho)
$$

Inserting this series expansion into Eq. 3 gives

$$
b_{n 0}=(2 n+2) \exp \left(\frac{i a}{2}\right) \sqrt{\frac{\pi}{a}} \times \sum_{s=0}^{\infty}(2 s+1) i^{s} J_{s+1 / 2}\left(\frac{a}{2}\right) \int_{0}^{1} R_{2 s}^{0}(\rho) R_{n}^{0}(\rho) \rho d \rho .
$$

Using the orthogonality of the Zernike radial polynomials, the expansion coefficients reduce to

$$
b_{n 0}=i^{n / 2} \exp \left(\frac{i a}{2}\right) \sqrt{\frac{\pi}{a}}(n+1) J_{(n+1) / 2}\left(\frac{a}{2}\right)
$$

Note that the Bessel function in Eq. 8 has a fractional order and, in general, a complex argument. These values, however, are readily calculated with mathematics packages such as MATLAB (MathWorks, Natick, MA), Mathematica (Wolfram, Champaign, IL), as well as coded with numerically stable algorithms.[6] When only defocus 
is present $a=-2 \pi W_{20}$ and the argument of the Bessel function in Eq. 8 is real. When $W_{20}>0$, the argument of the Bessel is negative and the identity $J_{v}(-x)=\exp (i \pi v) J_{v}(x)$ for positive $x$ needs to be used. When only apodization is present $a=i \alpha_{20}$ and the argument of the Bessel function in Eq. 8 is purely imaginary. In this case, the identity $J_{v}(i x)=\exp (i \pi v / 2) I_{v}(x)$, where $I_{v}(x)$ is the modified Bessel function of the First Kind is used. Both of these cases were examined in [1], but the expansion coefficients were calculated with a series expansion of the pupil function. This series expansion converges for small levels of defocus and apodization, but becomes numerically unstable for large values. These results leverage the numerically stable algorithms already developed for Bessel functions.

\section{Examples}

An interesting case not previously examined with this methodology is when defocus is combined with a Gaussian apodized pupil. Mahajan[7] provides a description of the OTF in this case. Fig. 1(a) compares the OTFs calculated with the linear expansion techniques describe here with numerical integration of Mahajan's expressions. In all cases, the defocus is $W_{20}=2 \lambda$ and the apodization value is $\alpha_{20}=\{1,4,9\}$. The modulation values between the two methods match to with $10^{-7}$. As expected, the Gaussian apodization suppresses the contrast reversal associated with the defocus and the OTF becomes more Gaussian-like with increased apodization.

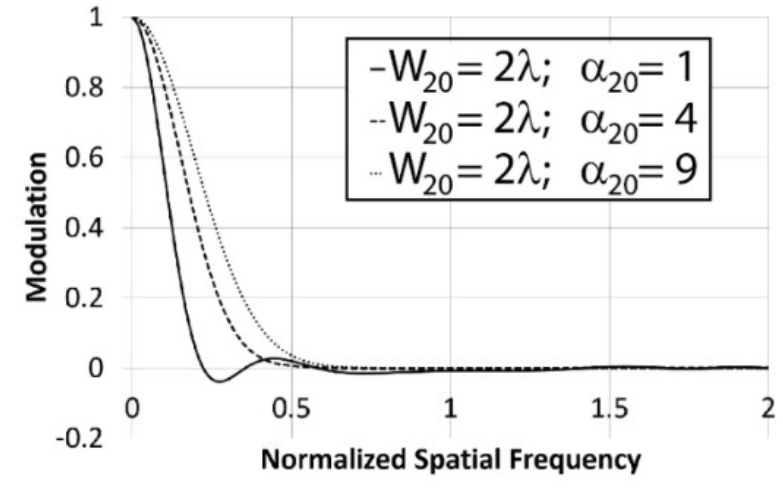

(a)

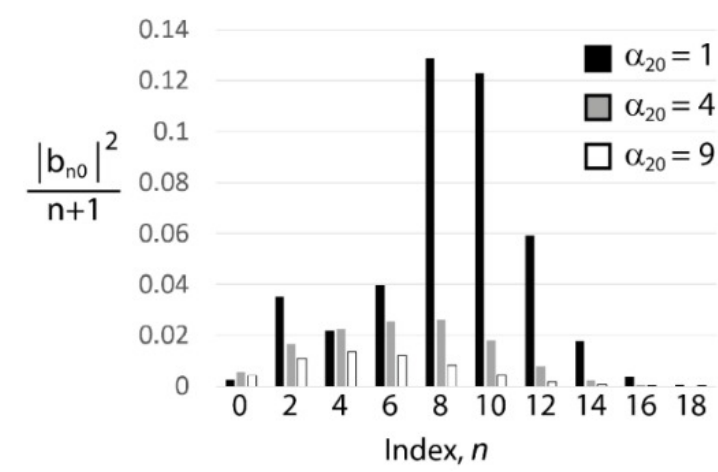

(b)

Fig. 1. (a) OTF predicted with a linear combination of G-Functions. (b) Terms of the convergence sum $c_{N}$ for different levels of apodization.

Eq. 5 is used to determine the relative transmission of the pupil. For the cases examined here, the transmissions are $0.432,0.125$ and 0.056 for apodization factors $\alpha_{20}$ of 1,4 and 9 , respectively. The sum $c_{N}$ will converge to these values when a sufficient number of expansion terms are used. Fig. 1(b) shows the individual terms of the sum $c_{N}$. For all cases, the pupil expansion converges after 10 terms. Fig. 2 also illustrates some of the utility of the OTF expansion technique. When $\alpha_{20}=1$, the terms in the figure are skewed to the right with a peak for the $n=8$ term. This suggests that higher order (i.e. more oscillatory) are needed to represent the OTF, as is to be expected based on the contrast reversal around $\beta=0.3$ seen in Fig. 1(a) for this case. Increasing the apodization factor has the effect of shifting the distribution of the $c_{N}$ terms to the left, meaning the OTF is progressively represented by smoother GFunctions and the overall OTF is improved. This improvement, however, comes at the expense of the overall transmission of the system which is reflected in the diminishing area under the $c_{N}$ terms with increased apodization.

An analytic expression for the expansion coefficients for a decomposition of a general quadratic pupil function is derived. The coefficients in turn enable a linear expansion of the OTF into a set of G-Functions. The coefficients relate the effects of defocus and Gaussian apodization directly to the components of the OTF. While most pupil functions assume a uniformly illuminated pupil, Gaussian apodization becomes relevant in systems illuminated with Gaussian beams; in the human eye, where the Stiles-Crawford effect is often modeled with Gaussian apodization[8]; or in systems requiring the suppression of diffraction rings.[9] The results also have application to systems with annular apertures and zone plates, as well as diffraction efficiency calculation in kinoforms.[10]

\section{References}

[1] Schwiegerling, J., "Relating wavefront error, apodization, and the optical transfer function: on-axis case,” J. Opt. Soc. Am. A 31, $2476-2483$ (2014).

[2] Schwiegerling, J., “Optical transfer function optimization based on linear expansions,” Proc. SPIE 9579, 95790H (2015).

[3] Schwiegerling, J., "Relating Wavefront Error, Apodization and the Optical Transfer Function: General Case” J. Opt. Soc. Am. A 34, 726-731

(2017).

[4] Born, M. and Wolf E., Principles of Optics, 6th ed. Permagon Press, London, 464-466 (1980). 
[5] Boivin, A., Théorie et Calcul des Figures de Diffraction de Révolution, Les Presses do L’Université Laval, 1964). 179 (1964).

[6] Press, W. H.,, Teukolsky, S. A., Vetterling, W. T., Flannery, B. P., Numerical Recipes: The Art of Scientific Computing, 3rd ed., Cambridge, United Kingdom, 283-292 (2007).

[7] Mahajan, V. N. “Gaussian apodization and beam propagation,” In: Progress in Optics, Vol. 49, Ed: E. Wolf (Elsevier B.V., Netherlands, 3134, (2006)

[8] van Meeteren, A., "Calculations of the optical modulation transfer function of the human eye for white light," Opt. Acta 21, 395-412 (1974).

[9] Jacquinot P., and Roizen-Dossier M. B., “Apodisation,” In: Progress in Optics, Vol. 3, Ed: E. Wolf, North Holland, Netherlands (1964).

[10] Schwiegerling J., "Diffraction efficiency and aberrations of diffractive elements obtained from orthogonal expansion of the point spread function,” Proc. SPIE 9953, 995307 (2016). 